\title{
Quality indicators for screening colonoscopies and colonoscopist performance and the subsequent risk of interval bowel cancer: a systematic review protocol
}

\author{
Martin Lund ${ }^{1}$. Mette Trads ${ }^{2,4}$. Rune Erichsen ${ }^{3} \cdot$ Berit Andersen ${ }^{1}$ \\ ${ }^{7}$ Department of Public Health Programmes, Randers Regional Hospital, Randers, Denmark, ${ }^{2}$ Danish Centre of Systematic Reviews: a Joanna Briggs \\ Institute Centre of Excellence, Aalborg Denmark, ${ }^{3}$ Department of Surgery, Section of Coloproctology, Aarhus University Hospital, Aarhus, Denmark, \\ Department of Clinical Epidemiology, Aarhus University Hospital, Denmark, and ${ }^{4}$ Department of Surgery, Randers Regional Hospital, Randers, \\ Denmark
}

Review question/objective: The objective of this systematic review is to assess the association between quality indicators related to the individual colonoscopist's performance and subsequent interval cancers in patients participating in bowel cancer screening programs, following the JBI approach. ${ }^{1}$

This systematic review of association will search all relevant literature on the subject to answer the following review questions:

1. Are the commonly used quality indicators of cecal intubation rate (CIR), adenoma detection rate (ADR), polyp recovery (PR), withdrawal time (WT), and incomplete adenoma resection (IAR)/incomplete polyp resection (IPR) associated with the outcome of interval cancer?

2. Is it possible to determine cut-off values that are significantly associated with each of the quality indicators mentioned above and the outcome of interval cancer?

Keywords Colonoscopy; endoscopist; interval cancer; quality indicator; screening

JBI Database System Rev Implement Rep 2017; 15(8):1991-1997.

\section{Background}

W orldwide, in 2012, colorectal cancer (CRC) was the third most common cancer in men (746,000 cases, representing $10 \%$ of the total cancer incidence) and the second most common cancer in women $(614,000$ cases, representing $9.2 \%$ of the total cancer incidence). ${ }^{2}$ Almost $55 \%$ of cases occured in more developed regions. Incidence rates vary ten-fold in both genders, worldwide. The highest estimated rates are in Australia and New Zealand (44.8 and 32.2 per 100,000 person-years in men and women, respectively), and the lowest rates are in Western Africa (4.5 and 3.8 per 100,000 personyears in men and women respectively). ${ }^{2}$ The probability of developing CRC in a lifetime is $4.6 \%$ (one in 22) for American men and $4.2 \%$ (one in 24) for American women. ${ }^{3}$ Between 2004 and 2013, the decline in CRC in the United States has been

Correspondence: Martin Lund, martlund@rm.dk

There is no conflict of interest in this project.

DOI: 10.11124/JBISRIR-2016-003241
$3 \%$ per year in both men and women. ${ }^{3}$ This decline most likely reflects the increased uptake of screening in the form of colonoscopy as the primary screening tool. Colonoscopy can prevent cancer by removal of precancerous lesions. ${ }^{3}$ In the United States, colonoscopy use among adults aged 50 years and older has tripled, from $21 \%$ in 2000 to $60 \%$ in $2015 .^{3}$

In 2012, a total of 694,000 individuals worldwide died from CRC (corresponding to $8.5 \%$ of all cancer-related deaths), with a higher number of deaths in the less developed regions of the world $(52 \%$ of all deaths from CRC), reflecting a poorer survival in these regions in comparison with the more developed regions of the world. ${ }^{2}$ The variability in mortality rates worldwide is six-fold in men and four-fold in women, with the highest estimated mortality rates in both sexes occurring in Central and Eastern Europe (20.3 per 100,000 person-years for men, 11.7 per 100,000 person-years for women), and the lowest estimated mortality rates in Western Africa (3.5 and 3.0 per 100,000 person-years for men and women, respectively). ${ }^{2}$ In the United States, the five-year 
relative survival rates (2006-2012), are 65\% for men and women with CRC. ${ }^{3}$

Bowel cancer screening programs can be structured in different ways. An organized screening program involves a systematic process of inviting a defined target population to participate. By contrast, opportunistic screening is delivered outside of an organized screening program on an $a d$ hoc basis. ${ }^{4}$ Bowel cancer screening programs can be based on invasive imaging techniques like colonoscopy or sigmoidoscopy as the primary screening tool, or non-invasive tests on stool samples by the use of the fecal immunochemical test for hemoglobin (FIT) or the guaiac fecal occult blood test (gFOBT) as the primary screening tool. The cut-off level of the FIT or the gFOBT determines the number of patients who will have a colonoscopy as the secondary screening tool. ${ }^{4}$

A recent review showed that primary screening colonoscopies/diagnostic colonoscopies, with removal of all detected lesions, were associated with a $61 \%$ reduction in CRC incidence (RR: 0.39; $95 \%$ confidence interval $[\mathrm{CI}]: 0.26-0.60)$ in patients with nonmalignant findings compared to patients from the same population who had no colonoscopy. ${ }^{5}$ The overall CRC mortality rate was also reduced, with 61\% (RR 0.39; 95\% CI: 0.35-0.43) in patients with non-malignant findings compared to patients from the same population who had no colonoscopy. ${ }^{5}$ The duration of follow-up in the 11 included studies varied, with three studies with more than 10 years of follow-up, seven studies with five to 10 years of follow-up, and one study with less than five years of follow-up. ${ }^{5}$

The quality of CRC screening is highly dependent on the quality of the colonoscopy, including the qualifications of the colonoscopist. Among the commonly used quality indicators in screening colonoscopies, the ADR rates as very important. ${ }^{6,7}$ In the setting of screening colonoscopy as the primary screening tool, one study found that patients examined by colonoscopists with an individual rate of adenoma detection of less than $20 \%$ had a significantly increased risk of interval CRC compared with patients examined by colonoscopists with a detection rate of $20 \%$ or more. ${ }^{6}$ The hazard ratio for an ADR of $20 \%$ or higher was compared with rates below $11 \%$ (hazard ratio, 10.94; 95\% CI, 1.37-87.01), 11.0-14.9\% (hazard ratio, 10.75; $95 \%$ CI, 1.36-85.06), and 15.0-19.9\% (hazard ratio, $12.50 ; 95 \% \mathrm{CI}, 1.51-103.43)(P=0.02$ for all three comparisons). ${ }^{6}$ Another study reported that the risk of interval cancer decreased approximately linearly with increasing ADRs. Thus, with the ADR modeled as a continuous variable, each $1 \%$ increase in the ADR predicts a $3 \%$ decrease in the risk of interval cancer (hazard ratio, 0.97; 95\% CI, 0.96-0.98). ${ }^{7}$

To monitor the quality of screening colonoscopies and set standards that should be met in order to justify the level of quality of a screening program, it is necessary to have the right tools. This systematic review of association aims to identify all literature assessing the association between the commonly used quality indicators in screening colonoscopies (either used as a primary screening procedure or after positive stool samples) and the outcome of interval cancer. In that context, this systematic review of association also aims to reveal whether it is possible to establish cut-off values that are significantly associated with each of the quality indicators commonly used in screening colonoscopies and the outcome of interval cancer in the same way that the cutoff value has been established for ADR.

A preliminary search for existing reviews on the topic was conducted. The sources searched were the JBI Database of Systematic Reviews and Implementation Reports, Cochrane Database of Systematic Reviews, CINAHL, PubMed, Embase, EPPI, and Epistemonikos. No review on the topic was identified. PROSPERO was also searched to identify possible protocols or unfinished reviews on the topic, but no ongoing reviews were found.

\section{Definitions}

\section{Cecal intubation rate (CIR)}

Defined as the percentage of screening colonoscopies in which the colonoscopist has achieved cecal intubation. Cecal intubation is defined as the introduction of the tip of the colonoscope into the cecal caput. In order to visualize the entire colonic mucosa, introduction of the colonoscope into the cecum is mandatory. Cecal intubation should be documented by naming and photographing cecal landmarks; i.e. the appendiceal orifice, ileocecal valve and/or terminal ileum. ${ }^{8,9}$

\section{Adenoma detection rate (ADR)}

Defined as the percentage of screening colonoscopies in which at least one adenoma is identified and removed per colonoscopy. 8,9 


\section{Polyp recovery (PR)}

Defined as the percentage of excised polypectomy specimens retrieved for histological analysis in each patient. ${ }^{10}$

\section{Withdrawal time (WT)}

Defined as the time from which the cecum is reached to when the colonoscope is withdrawn from the anus. ${ }^{9}$ Withdrawal time is only of interest in the setting of negative screening colonoscopies; i.e. screening colonoscopies with no pathology detected. ${ }^{10}$

\section{Incomplete adenoma resection (IAR)/incomplete polyp resection (IPR)}

Defined as the resection of an adenoma/polyp, with any of the lateral and/or deep margins involved with tumor cells. ${ }^{11}$

\section{Interval cancer}

Defined as a CRC “diagnosed after a colorectal screening examination or test in which no cancer is detected, and before the date of the next recommended exam". ${ }^{12(p .1263)}$ In the context of this systematic review of association, interval cancer is defined as a CRC diagnosed after a screening colonoscopy in which no cancer has been detected, and before the date of the next recommended exam.

The term postcolonoscopy CRC (PCCRC) is sometimes used instead of interval cancer. There is no standard definition for PCCRC. A PCCRC can be defined in different ways in different settings. In studies where the term PCCRC is used instead of interval cancer, we will analyze the PCCRC as interval cancer and evaluate the definition of the PCCRC in each case.

\section{Inclusion criteria}

\section{Types of participants}

This systematic review of association will consider studies that include persons, regardless of comorbidity, who have undergone a screening colonoscopy as participants in a bowel cancer screening program. Patients with known CRC, hereditary nonpolyposis colorectal cancer (HNPCC) or familial adenomatous polyposis (FAP) will be excluded.

In the context of this systematic review, the colonoscopists performing the screening colonoscopies will also be included as participants.

\section{Exposure of interest}

This systematic review of association will consider studies evaluating the following quality indicators: cecal intubation rate (CIR), adenoma detection rate (ADR), polyp recovery (PR), withdrawal time (WT), and incomplete adenoma resection (IAR)/incomplete polyp resection (IPR). These are commonly used quality indicators for screening colonoscopies in bowel cancer screening programs.

\section{Outcome}

This systematic review of association will consider studies that include the outcome of interval cancer, i.e. CRC diagnosed after a screening colonoscopy in which no cancer was detected, and before the date of the next recommended examination. In studies where the term PCCRC is used instead of interval cancer, we will analyze the PCCRC as interval cancer and evaluate the definition of the PCCRC in each case.

\section{Types of studies}

The quantitative component of this systematic review of association will consider epidemiological studies and prospective and retrospective cohort studies for inclusion. The quantitative component of the systematic review will also consider case series for inclusion.

Qualitative and economic components will not be included in this systematic review.

In the absence of research studies, no other articles such as opinion papers and reports will be considered.

\section{Search strategy}

The search strategy aims to identify both published and unpublished studies. A three-step search strategy will be utilized in this systematic review of association. An initial limited search of MEDLINE and CINAHL will be undertaken followed by an analysis of the text words contained in the titles and abstracts, and of the index terms used to describe the articles. A second search using all identified keywords and index terms will then be undertaken across all included databases. Thirdly, the reference lists of all high quality studies will be searched for additional studies. Studies published in English, German, Danish, Swedish, or Norwegian will be considered for inclusion in this review. Worldwide, 
the first opportunistic bowel cancer screening program, using gFOBT as the primary screening tool, was established in Austria in 1980. ${ }^{4}$ Therefore, studies published from 1980 onward will be considered for inclusion in this review.

The databases to be searched include: PubMed, Embase, Web of Science, and CINAHL.

The search for unpublished studies will include: SIGLE/EAGLE (www.opengrey.eu) and Grey Literature Report (www.greylit.org).

Initial keywords will be: "colonoscopy"; "screening"; "quality indicator"; "cecal intubation rate (CIR)"; "adenoma detection rate (ADR)"; "polyp recovery (PR)"; "withdrawal time (WT)"; "incomplete adenoma resection (IAR)/incomplete polyp resection (IPR)"; and "interval cancer".

\section{Preliminary search strategy}

We will search the databases MEDLINE (PubMed), Embase (Ovid), Web of Science and CINAHL (EBSCO) from 1980 to current date with language restrictions to English, German, Danish, Swedish, and Norwegian. The search for PubMed will be as follows: (/(()(/()(““Colonoscopy”[Mesh]) OR colonoscop* [Text Word] )) AND ((((“Mass Screening”'[Mesh]) OR "Early Detection of Cancer"[Mesh]) OR ((“Colorectal Neoplasms/diagnosis”[Mesh] OR "Colorectal Neoplasms/prevention and control"'[Mesh]))) OR screening*[Text Word])) AND

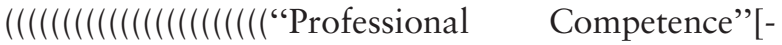
Majr]) OR caecal intubation[Text Word]) OR cecal intubation[Text Word]) OR adenoma detection rate $[$ Text Word]) OR adenoma miss rate*[Text Word]) OR cancer detection rate*[Text Word]) OR cancer miss rate* [Text Word]) OR polypectomy rate*$^{*}[$ Text Word] $)$ OR withdrawal time*[Text Word]) OR bowel preparation*[Text Word]) OR interval cancer*[Text Word]) OR post-colonoscopy colorectal cancer*[Text Word]) OR postcolonoscopy colorectal cancer*[Text Word]) OR PCCRC[Text Word]) OR safe sedation[Text Word]) OR patient comfort[Text Word]) OR neoplasia detection rate* [Text Word]) OR polyp recovery [Text Word]) OR polyp detection rate*[Text Word]) OR polyp miss rate*[Text Word]) OR adenoma resection[Text Word]) OR tumour resection[Text Word]) OR polyp resection[Text Word]))))) AND ((“'1980/01/01”[PDat]: “3000/12/31”[PDat]) AND (Danish[lang] OR English[lang] OR German[lang] OR Norwegian[lang] OR Swedish[lang]))))).
The search will be modified and searched in the other databases.

OpenGrey (www.opengrey.eu) and Grey Literature Report (www.greylit.org) will be searched with the initial keywords.

\section{Assessment of methodological quality}

Quantitative papers selected for retrieval will be assessed by two independent reviewers for methodological validity prior to inclusion in the review using the standardized critical appraisal instrument, JBI Critical Appraisal Checklist for Analytical Cross Sectional Studies (Appendix I). Any disagreements that arise between the reviewers will be resolved through discussion and consensus, or with a third reviewer as arbiter.

\section{Data extraction}

Quantitative data will be extracted from papers included in the review using the standardized data extraction tool developed for systematic reviews of association, amended to fit into this systematic review of association (Appendix II). The data extracted will include specific details about the study methodology, setting, primary screening tools, population, investigated quality indicator(s), outcome, statistical methods, and results of the statistical analysis of significance for the review questions and the specific objective of this systematic review of association.

Authors of primary studies will be contacted for clarification or missing information.

\section{Data synthesis}

Where possible, observational, quantitative papers will be pooled in statistical meta-analysis using the Joanna Briggs Institute System for the Unified Management,. Assessment and Review of Information (JBI-SUMARI). Effect sizes will be expressed as relative risk for epidemiological study designs, and prospective and retrospective cohort studies (for categorical data), and weighted mean differences (for continuous data) and their $95 \%$ CI will be calculated for the analysis. A random effects model will be used and heterogeneity will be assessed statistically using the standard Chi-square. Where statistical pooling is not possible, the findings will be presented in narrative form including tables and figures to aid in data presentation where appropriate. 
Descriptive, epidemiological study designs, including case series, will where possible be synthesized and presented in a tabular summary, with the aid of narratives and figures.

The data will be stratified depending on whether the data originates from bowel cancer screening programs with colonoscopy as the primary screening tool or from bowel cancer screening programs with, for example, FIT as the primary screening tool.

Studies including the predefined types of participants, quality indicator(s) and outcomes will be selected for inclusion. The critical appraisal instrument will divide the included studies into groups of high, moderate or poor quality, or not applicable quality on the basis of the possibility of bias in their design, conduct and analysis. Only studies for which the answer to all questions is "yes" in the critical appraisal instrument will be considered as high quality studies. A sensitivity analysis will be completed where only high quality studies are selected for statistical analysis. If the results of the sensitivity analysis are significantly different from the statistical analysis including all included studies, the results of the high quality studies will be emphasized as the main result in the review.

\section{Acknowledgements}

We would like to thank librarian Henrik Laursen, Viborg Regional Hospital, for drafting preliminary search strategies.

\section{References}

1. Moola S, Munn Z, Sears K, Sfetcu R, Currie M, Lisy K, et al. Conducting systematic reviews of association (etiology): The Joanna Briggs Institute's approach. Int J Evid Based Healthc 2015;13(3):163-9.
2. Colorectal Cancer, Estimated Incidence, Mortality and Prevalence Worldwide in 2012. 2012; Available at: http:// globocan.iarc.fr/Pages/fact_sheets_cancer.aspx?cancer= colorectal. Accessed May 8. 2016.

3. Siegel RL, Miller KD, Jemal A. Cancer Statistics, 2017. CA Cancer J Clin 2017;67(1):7-30.

4. Schreuders EH, Ruco A, Rabeneck L, Schoen RE, Sung JJ, Young GP, et al. Colorectal cancer screening: a global overview of existing programmes. Gut 2015;64(10): 1637-49.

5. Pan J, Xin L, Ma YF, Hu LH, Li ZS. Colonoscopy Reduces Colorectal Cancer Incidence and Mortality in Patients With Non-Malignant Findings: A Meta-Analysis. Am J Gastroenterol 2016;111(3):355-65.

6. Kaminski MF, Regula J, Kraszewska E, Polkowski M, Wojciechowska U, Didkowska J, et al. Quality indicators for colonoscopy and the risk of interval cancer. N Engl J Med 2010;362(19):1795-803.

7. Corley DA, Jensen CD, Marks AR, Zhao WK, Lee JK, Doubeni $\mathrm{CA}$, et al. Adenoma detection rate and risk of colorectal cancer and death. N Engl J Med 2014;370(14):1298-306.

8. Pullens HJ, Siersema PD. Quality indicators for colonoscopy: Current insights and caveats. World J Gastrointest Endosc 2014;6(12):571-83.

9. Atia MA, Ramirez FC, Gurudu SR. Quality monitoring in colonoscopy: Time to act. World J Gastrointest Endosc 2015;7(4):328-35.

10. Valori R, Patnick J, Coleman L, Winder R, Campbell D, Barton $\mathrm{R}$, et al. Quality assurance guidelines for colonoscopy, NHS BCSP Publication No 6. 2011; Available at: https://www. gov.uk/government/uploads/system/uploads/attachment data/file/427591/nhsbcsp06.pdf. Accessed March 20, 2016.

11. Lee SP, Sung IK, Kim JH, Lee SY, Park HS, Shim CS. Risk factors for incomplete polyp resection during colonoscopic polypectomy. Gut Liver 2015;9(1):66-72.

12. Sanduleanu S, le Clercq CM, Dekker E, Meijer GA, Rabeneck $\mathrm{L}$, Rutter MD, et al. Definition and taxonomy of interval colorectal cancers: a proposal for standardising nomenclature. Gut 2015;64(8):1257-67. 
Appendix I: Critical appraisal instrument

JBI critical appraisal checklist for analytical cross sectional studies

Reviewer Date

Author Year Record Numbe Yes No Unclear Not applicable

1. Were the criteria for inclusion in the sample clearly defined?

2. Were the study subjects and the setting described in detail?

3. Was the exposure measured in a valid and reliable way?

4. Were objective, standard criteria used for measurement of the condition?

5. Were confounding factors identified?

6. Were strategies to deal with confounding factors stated?

7. Were the outcomes measured in a valid and reliable way?

8. Was appropriate statistical analysis used?
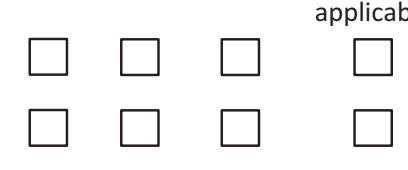

$\square \quad \square \quad \square \quad \square$

$\square \quad \square \quad \square \quad \square$

$\square \quad \square \quad \square \quad \square$

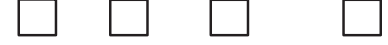

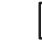
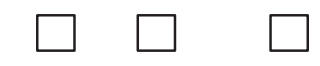

Overall appraisal: $\quad$ Include $\square$ Exclude $\square$ Seek further info 


\title{
Appendix II: Data extraction instrument
}

\author{
JBI data extraction form - etiology \\ Reviewer: \\ Author: \\ Journal: \\ Date \\ Year \\ Record Number
}

Study Method : Longitudinal

Retrospective

Observational

Other

Unclear

Participants

Setting

Primary screening tool

Population

Quality indicators investigated (cross mark $\mathrm{x}$ )

Cecal intubation rate $(\mathrm{CIR})$ :

Adenoma detection rate (ADR):

Polyp recovery (PR):

Withdrawal time (WT):

Incomplete adenoma resection (IAR)/incomplete polyp resection (IPR):

Outcome: interval cancer

\begin{tabular}{|l|l|}
\hline Quality indicator & Statistical method \\
\hline Cecal intubation rate (CIR): & \\
\hline Adenoma detection rate (ADR): & \\
\hline Polyp recovery (PR): & \\
\hline Withdrawal time (WT): & \\
\hline $\begin{array}{l}\text { Incomplete adenoma resection (IAR)/incomplete } \\
\text { polyp resection (IPR): }\end{array}$ & \\
\hline
\end{tabular}

$\underline{\text { Results of statistical analysis }}$ 